\title{
Development and Application of Big Data in the Field of Satellite Navigation
}

\author{
Jianjun Zhang $\mathbb{D}^{1,2}$ and Jing $\mathrm{Li} \mathbb{D}^{3}$ \\ ${ }^{1}$ Beijing Institute of Spacecraft System Engineering, Beijing 100094, China \\ ${ }^{2}$ China Academy of Space Technology, Beijing 100094, China \\ ${ }^{3}$ Beijing Institute of Technology, Beijing 100081, China \\ Correspondence should be addressed to Jing Li; bitljing@163.com
}

Received 22 April 2020; Revised 10 November 2020; Accepted 4 July 2021; Published 19 July 2021

Academic Editor: Fuhong Lin

Copyright (C) 2021 Jianjun Zhang and Jing Li. This is an open access article distributed under the Creative Commons Attribution License, which permits unrestricted use, distribution, and reproduction in any medium, provided the original work is properly cited.

\begin{abstract}
Based on the characteristics of big data, the meaning of fog computing, and the spatiotemporal data characteristics of satellite navigation systems, the concept, connotation, and characteristics of Beidou big data were put forward. The Beidou big data processing process was sorted out, the preliminary architecture of the Beidou big data system with fog computing function was built, and finally the fog computing based Beidou big data system was proposed. Big data research content provides technical support for further tapping the value of Beidou satellite navigation system data and realizing the incremental development of satellite navigation systems.
\end{abstract}

\section{Introduction}

Since the concept of "big data" was first put forward in the article "big data: Science in the petabyte era" in the special issue of nature in September 2008, big data has rapidly become a research hotspot in the global academic and industrial circles within a few years [1]. However, there is not a unified and recognized definition for big data. Generally speaking, the narrow sense of big data refers to the data set that takes more time to capture, manage, and process data than can be tolerated by using common software tools. The broad sense of big data includes not only the above content, but also the theory, method, and technology of processing big data, that is, it is more economical from high frequency and large new generation architecture, basic theory, and key technology designed to gain value from data of different structures and types with capacity. The typical characteristics of big data are volume, variety, velocity, veracity, and value [2].

Through opening, sharing, and collaboration, big data can mine new information and knowledge with huge imagination space, which can realize the continuous value-added of traditional applications and services and is highly valued. McKinsey believes that data has penetrated into every industry and business function area and become an important production factor. People's mining and application of massive data indicate the arrival of a new wave of productivity growth and consumer surplus [3].

Big data is characterized by large volume, diverse structure, and strong timeliness. As a new trend in the field of information technology, big data technology is quite different from traditional information technology in thinking and technology system. The processing of big data needs to adopt new computing architecture and intelligent algorithm and other new technologies; the application of big data emphasizes the application of new ideas to assist decision-making and discover new knowledge. Big data is not only "big," but also "new," which is a combination of new resources, new tools, and new applications.

At present, the concept of big data has gradually become popular. At home and abroad, all walks of life attach great importance to the development of big data and related basic theory and technology research and closely combined with various application fields [4] have set up major projects and 
supporting policies to promote the development of big data. There are various typical applications such as traffic big data, financial big data, and medical big data.

Satellite navigation system can provide all-weather, allweather, high-precision positioning, navigation, and timing services is an important spatial information infrastructure of the country. All major aerospace countries in the world attach great importance to the construction, application, and development of satellite navigation systems. China has completed the construction of the "Beidou- 1 " test navigation system and the "Beidou-2" regional navigation system. The "Beidou-3" global system is steadily advancing. The nextgeneration "Beidou-4" system is in urgent need of innovation and development. Carrying out the construction of Beidou satellite navigation system has become the focus of urgent research. In the future, the construction of satellite navigation system is the demonstration of the integration of heaven and earth. Big data expands the innovation connotation of satellite navigation system and innovates the design method of satellite navigation system. Big data enables the designers of satellite navigation system to improve the design quality and level with more quantitative means. Under the background of big data, the design, construction, operation, management, and application of Beidou satellite navigation system will fundamentally change the thinking pattern of single subsystem design, construction, and management and promote the innovation of system engineering theory, equipment system design, operation management mode, and application way in the direction of adapting to the requirements of the integration of heaven and earth under the background of development of big data.

However, due to the large amount of satellite data, it is necessary to design a new computing paradigm to meet the demand of data transmission and processing in the case of big data. To reduce the time delay and solve the problems caused by big data, fog computing is introduced in this paper into the Beidou satellite navigation system. Fog computing is considered as a server operating at the edge of the satellite network, offering special services that require network context information, location awareness, and ultralow latency, to help to promote the performance of the whole Beidou satellite navigation system.

The whole paper is organized as follows. The meaning of fog computing is given in Section 2. Section 3 introduces the development of Beidou satellite navigation system. The connotation and characteristics of big data in Beidou are discussed in Section 4. Section 5 gives out the challenges and deficiencies of Beidou big data, and it is concluded in Section 7.

\section{The Meaning of Fog Computing}

Fog computing is a distributed and open platform that integrates core capabilities of network, computing, storage, and applications on the edge of the network close to the source of things or data. It provides intelligent services nearby to meet the needs of industry digitalization in agile connection, real-time business, data optimization, and application. Key requirements for intelligence, security, and privacy protection and the characteristics of fog computing are as follows: (i) Connectivity. Connectivity is the foundation of fog computing. The diversities of connected physical objects and application scenarios require fog computing need to have rich connection functions, such as various network interfaces, network protocols, network topologies, network deployment and configuration, and network management and maintenance. Connectivity needs to fully learn from and absorb advanced research results in the network field, such as TSN, SDN, NFV, Network as a Service, WLAN, NB-IoT, and 5G, while also considering interconnection with various existing industrial buses.

(ii) The First Entry of Data. As a bridge from the physical world to the digital world, fog computing is the first entrance to data. It has a large amount of realtime and complete data. It can manage and create value based on the full life cycle of the data, which will better support predictive maintenance, asset efficiency, and innovative applications such as management and management; at the same time, as the first entry point for data, fog computing also faces challenges such as data real-time, certainty, and diversity.

(iii) Constraint. Fog computing products need to be adapted to the relatively harsh working conditions and operating environments of industrial sites, such as antielectromagnetic, dust-proof, explosion-proof, antivibration, and anticurrent/voltage fluctuations. In the industrial interconnection scenario, there are also higher requirements for the power consumption, cost, and space of fog computing devices.

(iv) Distribution. The actual deployment of fog computing is naturally distributed. This requires fog computing to support distributed computing and storage, realize dynamic scheduling and unified management of distributed resources, support distributed intelligence, and possess distributed security capabilities.

(v) Fusion. As a key bearer for the integration and coordination of operation technology and information and communication technology, fog computing needs to support coordination in connection, data, management, control, application, security, etc.

\section{Development of Beidou Satellite Navigation System}

3.1. Development Stage of Beidou Satellite Navigation System. The Beidou satellite navigation system (hereinafter referred to as the Beidou system) is a satellite navigation system that China builds independently and operates independently with a view to national security and economic and social development needs. It provides global users with all-weather, allweather, high-precision positioning, navigation, and important national space infrastructure for time service. In the late 
20th century, China began to explore the development path of satellite navigation systems suitable for national conditions and gradually formed a three-step development strategy [4-9].

The first step is to build the Beidou-1 satellite navigation system (also known as the Beidou satellite navigation test system). In 1994, the Beidou-1 satellite navigation system project was started; in 2000, two geostationary satellites were launched, the system was built and put into use, and the active positioning system was adopted to provide positioning, timing, wide area differential, and short report for Chinese users' communication service. In 2003, the third geostationary satellite was launched to further enhance system performance.

The second step is to build the Beidou-2 satellite navigation system. In 2004, the construction of Beidou-2 satellite navigation system project was started; at the end of 2012, 14 satellites (5 geostationary orbit satellites, 5 tilted geosynchronous orbit satellites, and 4 medium-circular earth orbit satellites) were launched. On the basis of compatibility with Beidou-1 technical system, Beidou-2 system adds passive positioning system to provide positioning, speed measurement, timing, wide area differential, and short message communication services for users in the Asia-Pacific region.

The third step is to build the Beidou global system. In 2009, the Beidou global system construction was started, inheriting Beidou active service and passive service two technical systems; plan to provide basic services for the countries along the "Belt and Road" and surrounding countries in 2018; around 2020, complete 30 satellites (3 geostationary satellites, 3 tilted geostationary orbit satellites, and 24 medium-circular earth orbit satellites) launched a network to provide services to users around the world.

3.2. Beidou-3 Satellite Navigation System. The Beidou-3 global satellite navigation system is based on the Beidou-2 regional satellite navigation system and uses a hybrid constellation composed of "3GEO+3IGSO+24MEO" satellites to improve the performance and measurement accuracy of the spaceborne atomic clock through the improvement of the navigation signal system, as shown in Figure 1. Interlink other technologies to achieve the goals of global service, performance improvement, business stability, and interoperability with other systems. At the same time, we must ensure the smooth transition of the Beidou-2 feature service and the regional system.

The Beidou-3 global system will provide continuous, stable, and reliable RNSS services worldwide and provide services such as RDSS, location report/short message communication, satellite-based enhancement, and power enhancement in China and surrounding areas. Global positioning accuracy will achieve the requirements of a level better than 4 meters and a height better than 6 meters, as shown in Figure 2.

On the basis of inheriting and reserving part of the navigation signal components of beidou- 2 system, a new navigation signal system design is adopted for the downlink navigation signal of Beidou-3 navigation satellite, which is characterized by signal spectrum separation, pilot, and data orthogonal. The design optimizes and adjusts the power ratio of signal components, improves the equivalent omnidirectional radiation power (EIRP) value of the downlink signal, and realizes the signal anti-interference. The significant improvement of capability and ranging accuracy improves the performance of navigation signal and the utilization efficiency, compatibility, and interoperability of navigation signal. At the same time, the satellite system has the ability of downlink navigation signal system reconstruction, which can be further upgraded and improved according to the future development and technological progress.

Beidou-3 system has established a stable and reliable intersatellite link. Through intersatellite link ranging and timing, it can realize multisatellite measurement, increase observation, improve the geometry observation structure of autonomous orbit determination, use the intersatellite measurement information to autonomously calculate and modify the orbit position and clock system of the satellite, realize the joint precise orbit determination of the satellite and the earth, and support the improvement of the precision of satellite orbit determination and time synchronization To improve the positioning and service accuracy of the whole system. Through intersatellite and satellite ground links, the monitoring and injection functions of overseas satellites are realized, and the "one-stop measurement and control" management of overseas satellites is realized.

At the same time, Beidou- 3 satellite adopts the information fusion technology of interactive support. During the normal operation of the satellite in orbit, the s-frequency point is used to realize the satellite to ground measurement and control, the l-frequency point is used to realize the time comparison between the satellite and the ground, the upstream injection operation control management of RNS service, and the Ka frequency point is used to realize the intersatellite ranging and communication. After combining and analyzing the information flow of the whole satellite, adopting the design of on-board information fusion, the information channel between the links with relatively independent functions is opened. Through the network protocol agreement, the information interactive backup between the s, 1 , and Ka frequency points of the satellite is realized, the uplink capability of the satellite is expanded, and the system reliability is improved. The comparison between Beidou satellite navigation system and foreign satellite navigation system is shown in Table 1.

\section{Connotation and Characteristics of Big Data in Beidou}

Beidou satellite navigation system in China attaches great importance to the accumulation of data. At present, Beidou data has reached a certain scale. The storage format of data is mainly structured data. The amount of unstructured data such as video and pictures is also increasing. Beidou satellite navigation system in China implements modular management, mainly including space segment, ground control segment, and user segment. It has the characteristics of crossregional network, complex technical composition, various departments, and wide business application. Therefore, 


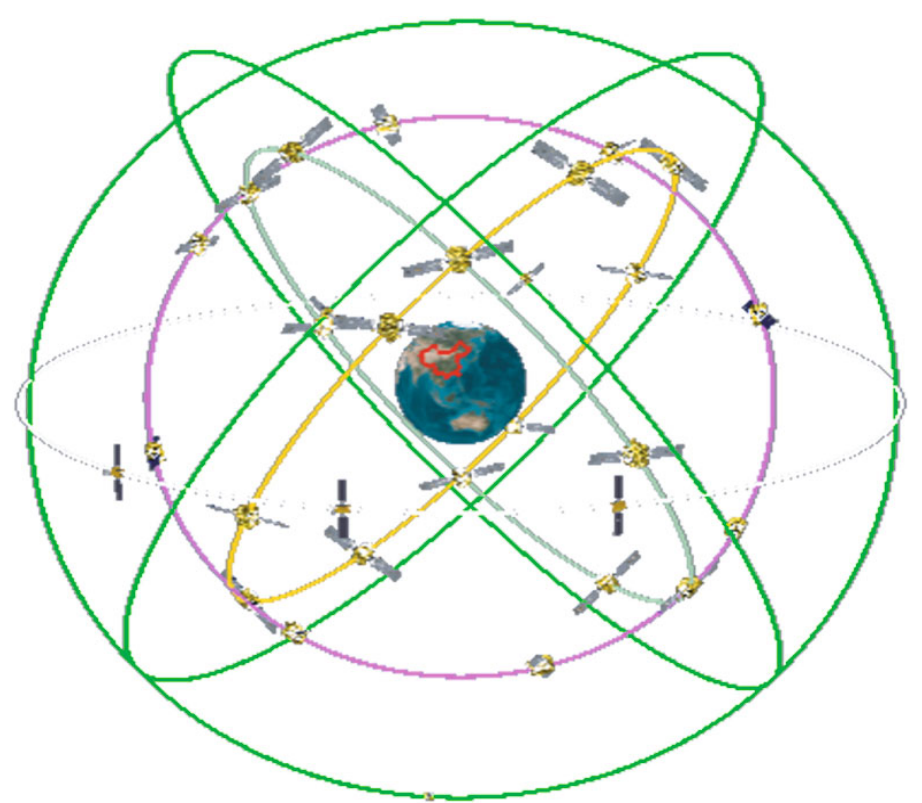

FIgURE 1: Schematic diagram of the Beidou-3 satellite constellation.

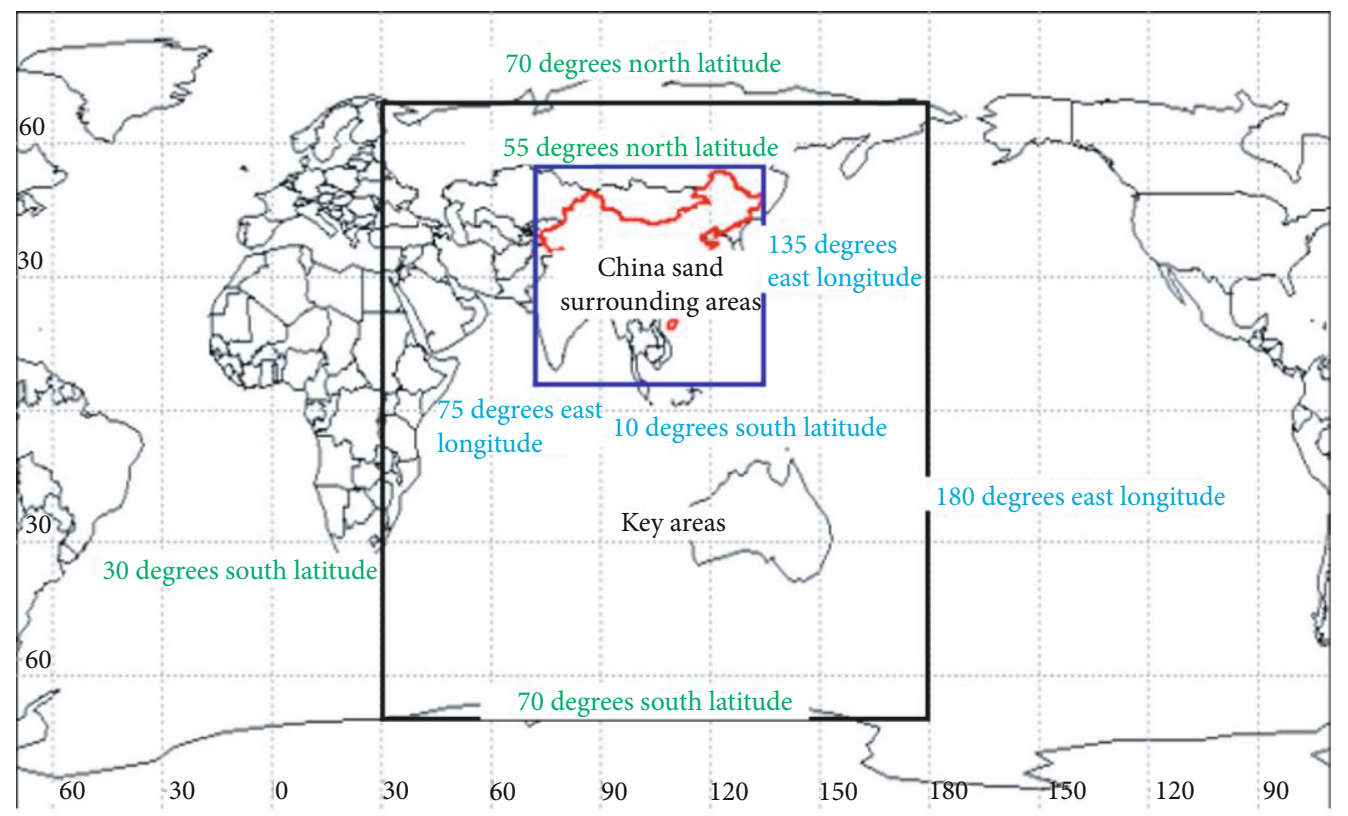

FIgure 2: Beidou-3 system service area.

Beidou satellite navigation system is a complex system engineering of full flow, full business, and full data. Beidou big data construction must be considered. We should strengthen top-level design, cross-department collaboration, crossbusiness management, and cross-technology coupling.

4.1. Connotation of Big Data in Beidou. With the steady progress of Beidou satellite navigation system, the gradual realization of the digitalization of navigation satellite load, the upgrading and transformation of ground telemetry and remote control [10], the real-time monitoring and the construction and application of a large number of information management systems serving various specialties are gradually achieved, and the scale and types of data are growing rapidly. These data together create big data of Beidou.

According to the different stages of data sources, big data of Beidou can be divided into big data of Beidou design, big data of Beidou construction, big data of Beidou operation management, and big data of Beidou application.

(1) Big Data in Beidou Design. With the rapid development of global satellite navigation system, the design of Beidou satellite navigation system is gradually evolving. Big data in Beidou design is also a process 


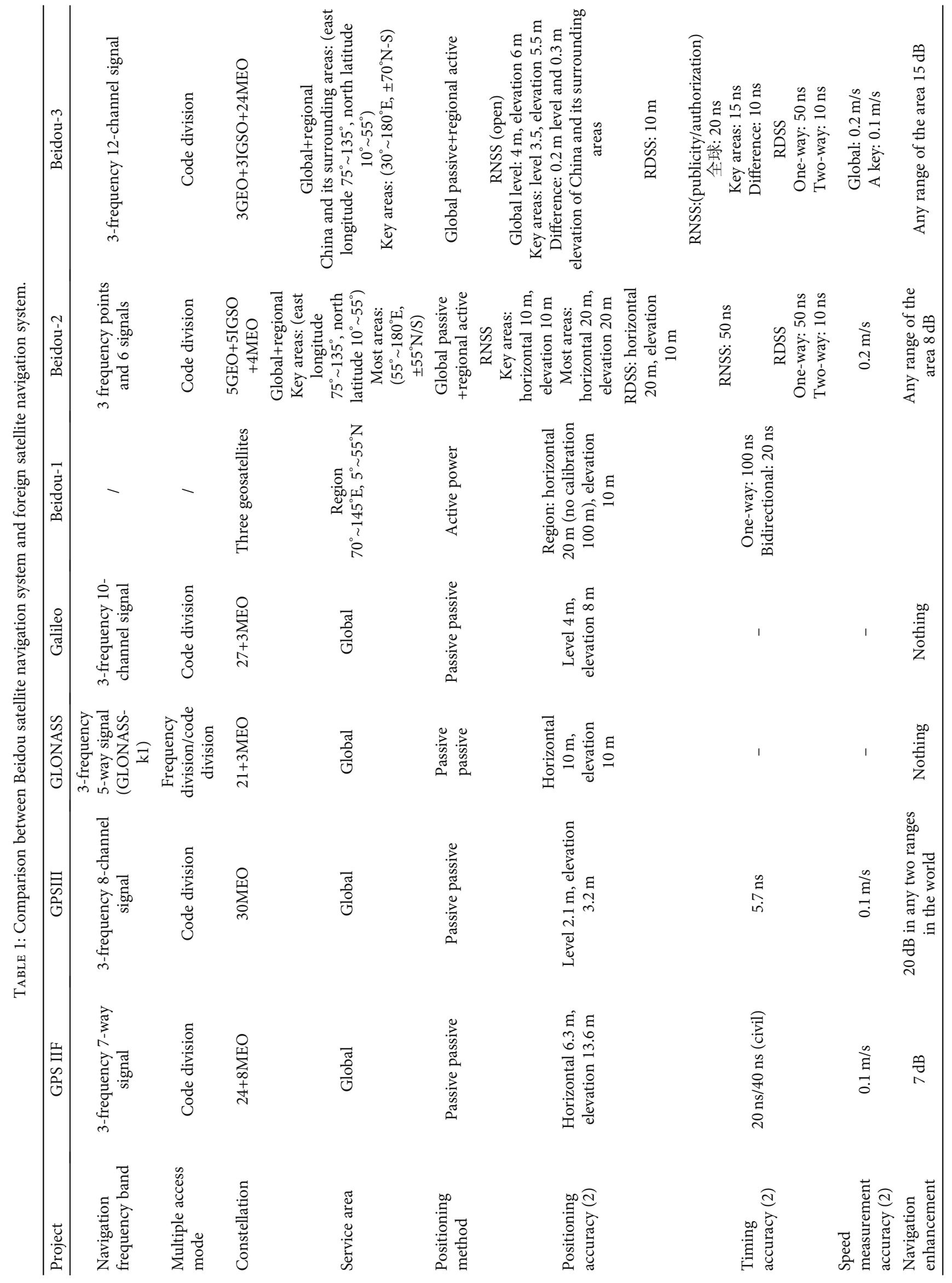




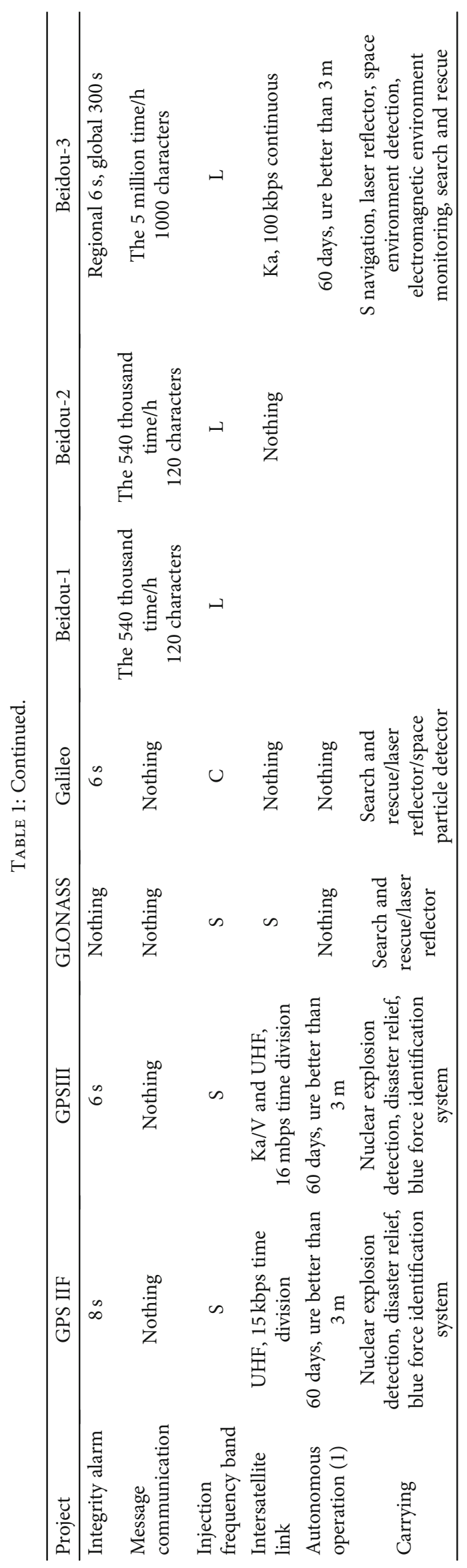


of gradually deepening the content. From the active design of "Beidou No.1," the regional active/passive design of "Beidou No.2," and the global active/passive design of "Beidou No.3," the design concept conforms to the national conditions of our country. Big data generated in the design embodies the wisdom of Beidou people, which is a valuable wealth of our country.

(2) Big Data in Beidou Construction. In 2004, the construction of Beidou satellite navigation system was officially launched. For the first time, it began to develop and produce satellites and launch vehicles in batch, launch in dense network, and explore new development mode of aerospace industry. We will tackle key technical problems and strive for sustainable development. It laid a foundation for Beidou construction and sustainable development. In Beidou project construction, technical research, and engineering practice, a large amount of structured, semistructured, and unstructured data has been generated through software and hardware access, data sharing, and other means in many key technologies such as onboard atomic clock, high-precision satellite to ground time comparison, monitoring receiver, and user terminal, as well as in the fields of system joint debugging and test, system launch, and so on.

(3) Big Data in Beidou Operation Management. The Beidou space segment is composed of multiple satellites, whose main task is to provide navigation services to all kinds of navigation users. The data produced by satellites are mainly telemetry information and navigation message information. Telemetry information can comprehensively describe the operation status and real-time working parameters of all key equipment of the satellite. Navigation message is a kind of navigation service information broadcast to users by satellite. The user segment includes various types of user terminals, mainly receiving satellite navigation signals and finally realizing navigation, positioning, timing, and other applications. The user terminal mainly generates registration management information, positioning and timing processing result information, operation condition information, and other data. Beidou ground control center and several ground stations jointly complete the operation management and control of satellites in orbit. The ground control center mainly produces satellite ground, intersatellite and interstation measurement information, navigation business processing results, uplink injection information, operation monitoring information, management control information, and other data; the ground station mainly produces satellite navigation signal observation data, navigation message information, meteorological observation information, operation monitoring information, video, and other data.

(4) Big Data in Beidou Application. Beidou big data is the key element of Beidou value-added services. Using
Beidou navigation data can provide users with more rich value-added services and can also be an important reference for urban development and investment decision-making. For example, by improving the "travel map," it can more accurately reflect the regional economic situation and the travel habits of various groups, which can provide the basic basis for urban and infrastructure construction planning.

According to different sources of Beidou big data, the abovementioned Beidou big data is divided into two categories: one is internal data of Beidou; the other is external data. Internal data relates to space datum maintenance data, spaceborne time-frequency long-term evolution data, space atomic frequency standard transmission data, intersatelliteground link data, ground remote control telemetry data, intersatellite time transmission data, etc. External data involves earth orientation parameters, ionospheric spacebased real-time monitoring data, pulsar large-scale spacetime information data, multifrequency source time reference collaborative data, etc. These data are scattered in different places, managed by different units/departments, with scattered placement 3 . The characteristics of distributed management are as reference [11]. These data are not completely independent, they are interrelated and influence each other, and there is a relatively complicated relationship. For example, the earth's rotation conditions and ionospheric data will affect the operation and management of the satellite, and the operation and management will affect the accuracy of positioning and then provide a basis for the decision-making of the relevant departments. In addition, these data structures are complex and diverse. In addition to traditional structured data, they also contain a large amount of semistructured and unstructured data, and the sampling frequency and life cycle of these data are also different, from microseconds to minute level, even to the annual level.

Beidou big data should also include data resources generated by political, economic, scientific, technological, diplomatic, and educational activities related to Beidou system, as well as the general term for Beidou big data technology and all application system data under its support. All these integrate the definition of big data and the application requirements of Beidou system and represent the main connotation of Beidou big data.

From a deeper perspective, Beidou big data has changed from traditional report analysis and data statistics to intelligent, precise, networked, and collaborative and from traditional methods of structured data analysis to intelligent methods of unstructured data, the transformation of limited data into multidimensional, multigranularity, multimodel, and multimodal massive data. Beidou big data is a cross-business, cross-department, cross-region, and cross-professional of the entire industrial chain of Beidou design, Beidou construction, Beidou operation, and Beidou management. It is an effective tool for absorbing the value of Beidou data, promoting the development of Beidou and accelerating the leap-forward development of Beidou. It is an inevitable process for Beidou to move from shouldering foreign satellite navigation systems to leading the development of foreign satellite navigation. 
4.2. Features of Big Data. Beidou big data has the general characteristics of big data, that is, Beidou big data also has typical $5 \mathrm{~V}$ characteristics, mainly reflects in the following:

(1) Volume. With the completion of Beidou global satellite navigation system, Beidou data has reached $\mathrm{PB}$ scale. From the perspective of the operation of the Beidou system alone, the Beidou satellite navigation system is an all-weather long-term continuous operation system that generates at least $1 \mathrm{~TB}$ of operating data in 7 days. As the operating time continues, the scale of accumulated data becomes even larger.

(2) Variety. Beidou data is divided into spatial segment data, ground segment data, and user segment data according to the data source. The data generated by the satellite is mainly telemetry information and navigation message information; the ground control center mainly generates satellite-ground, intersatellite and interstation measurement information, navigation service processing results, upstream injection information, operation monitoring information, management control information and other data ground station. It mainly produces satellite navigation signal observation data, navigation message information, meteorological observation information, operation monitoring information, video, and other data.

According to the composition and operating characteristics of the Beidou system, the system data involves computer, geographic mapping, network communication, mechanical electrical, and other fields of information. There are many types of data but not complicated. Most of them are structured data and semistructured data. Unstructured are fewer data types.

(3) Velocity. Velocity of various observation information collections, data transmission, business processing, injection information generation, navigation message broadcast, and other aspects of the Beidou system has strong real-time requirements. On the other hand, Beidou navigation is directly related to target strike positioning and damage effect evaluation. The timing of operations is fleeting, and the real-time requirements of Beidou big data processing far exceed that of other civilian big data.

(4) Accuracy. The accuracy of the Beidou system operation and the performance of providing services to the outside world will directly affect the national security and the daily life of the people.

(5) Big Value. On the one hand, Beidou big data is used in combat command, vehicle transportation, power distribution transmission, and logistics support. Its value is self-evident. On the other hand, because the Beidou satellite navigation system data has a clear meaning and the relationship is basically clear, the value density is high.

In addition to $4 \mathrm{~V}$ characteristics, Beidou big data also has its own unique characteristics:

(1) Basic. The spatiotemporal relationship is to establish the position and time label of people, things, objects, and places under the unified spatiotemporal reference and their association. The time information and position information generated by the Beidou system are the basis of the time and position sequence of space things.

(1) Location Information Data. In the construction of the spatial foundation, each node in the system is physically related to the spatial location information.

(2) Time Information Data. In the system operation and maintenance, system information transmission, time synchronization design, and guarantee are required, and the information data in the system is related to time.

(2) Value-Added. Big data is not only related to the operation and management of Beidou itself, but also involves many aspects such as military confrontation, resource allocation, power transmission, and public management. The value-added services of Beidou data will become an important part of the economic development of the country in the future. The application prospects of Beidou big data will become broader as it cross-links with different industries.

(3) Confidentiality. The Beidou signal takes two forms: one is the authorization signal, and the second is the unauthorized signal, and the authorization signal is the military signal. The data directly relates to national defense security, and it is more reliable. The data confidentiality is different from big data in other industries.

(4) Confrontation. The opposing sides conduct navigation warfare, and navigation confrontation is manifested in combat command, intelligence reconnaissance, and weapon control. Navigation signal power enhancement and navigation signal spoofing are all reflected in Beidou big data.

\section{Challenges and Countermeasures of Beidou Big Data}

5.1. Challenges and Deficiencies of Beidou Big Data. At present, externally, the US GPS system uses technological advantages to form a leading navigation data technology, thereby forming a military advantage; internally, the national economy puts forward higher processing requirements for the big data generated by the Beidou system. Big data's higher requirements are specifically reflected in the following: 
(1) Beidou Strategy. Inheriting the previous experience and knowing yourself and others, the more fully the basis for the Beidou strategy formulation.

(2) System Design. Based on big data technology, the entire process of Beidou index design-test-assessment-improvement-confirmation is rapidly formed, which supports the rapid iteration of Beidou system design.

(3) System Operation Management. The real-time monitoring and analysis of Beidou big data quickly forms the basis for decision-making and forms the system of autonomous flight, autonomous management, and self-closed loop.

(4) System Application. Beidou big data realizes the sharing of information in the whole society through the electronic guide service, which meets the broad "navigation" needs of users' work, life, and entertainment to the greatest extent. Realize satellite navigation application services with true user guidance, information sharing, and network services.

(5) In terms of political thinking, adapt to the new requirements of the new era and play the unique role of the spatial and temporal characteristics of Beidou big data in ideological and political education and public opinion tracking

Faced with new requirements, the application of Beidou big data technology is also facing the following four challenges.

(1) Insufficient Use of Data Sharing. Beidou has accumulated massive amounts of data in its operation management. The Beidou satellite navigation system generates at least about $1 \mathrm{~TB}$ of operating data in 7 days, including all kinds of observation information, data transmission, business processing, ephemeris information, and navigation messages, which need to be processed in real time. However, the data organization is relatively scattered, and the existence of different business departments lacks strict unified standards, which makes data sharing difficult.

(2) There is a shortage of data analysis and processing. Due to the large data volume and fast data generation speed of the Big Dipper Big Data, the consistency, accuracy, and completeness of processing Big Dipper data lack proper and effective analysis and processing technology, and the data is not useful. Due to processing technology and other reasons, the current data can be truly effectively processed and thus realize value, account for only a small amount, and it is difficult to truly realize the value of data

(3) The value of data quality is insufficient. The sources of Beidou's massive data are diverse, and the data models are very different, such as relational data, XML data, graph data, streaming data, scalar data, vector data, and uneven quality, and $1 \%$ to $30 \%$ data have various delays, errors, and difficulty in processing integration, which make it difficult to achieve high-quality data acquisition and precise integration and then find difficulties in the evolution of data

(4) Insufficient Discrimination of Data. There are a lot of fake data in Beidou big data, which mainly consider the intentional interference of navigation reception. Its characteristics are mainly to make the false satellite signal stronger than the real navigation signal in the form of ensuring the normal operation of the reception, thereby calculating the wrong location information, and the authenticity of these data has a significant impact on the decision-making process and results.

5.2. Responses to Beidou Big Data. To solve the insufficiency of Beidou big data sharing use, insufficiency in analysis and processing, insufficiency in quality value, and insufficiency in authenticity, we can analyze from the key technologies involved in the life cycle of big data technology, mainly including system big data technology. The top-level design involves big data collection and preprocessing, big data storage and management, big data mining, etc. The main ways are as follows:

\section{(1) Top-level design of Beidou big data system}

Provide services for the design, construction, operation management, and application of Beidou satellite navigation system, plan the storage and use of Beidou big data, establish the overall framework of Beidou big data system, and build Beidou big data slave data according to the life cycle of big data technology. Collect all the processes of the last application.

(2) Acquisition and integration of high-quality Beidou big data

Big data acquisition and integration refers to acquiring data from Beidou data sources or sensors and processing and integrating them into data collections stored in computing systems. High-quality big data acquisition and integration refers to the process of big data acquisition and integration that maximizes data availability. In Beidou data integration, the quality of data sources will greatly affect the availability of integrated data. How to determine and select high-quality data sources to make them the source of data acquisition is the key issue to obtain high-quality integrated data.

Pay attention to the following issues during the process of acquiring and integrating big data: (1) data cleaning. Focus on noise data and irrelevant data in the data source; (2) normalize the expression and consistency of the data through data extraction technology; (3) data transformation is to simplify the data through normalization, rotation, projection, and other operations of data characteristic representation; (4) evaluate data quality effectively, consistency, correctness, 
completeness, and minimumness which are the basic indicators of data quality evaluation.

(3) Open, flexible, and scalable distributed Beidou big data storage technology

The traditional database technology is mainly based on relational data management, and the data size is relatively small. When facing the torrent of semistructured and structured Beidou big data, scalability has obstacles and it is difficult to meet the needs. Abandon the traditional data storage design and build a developed, flexible, and scalable distributed Beidou data storage management system based on the Hadoop platform. Carry out a comprehensive sorting, classification, and integration of the navigation system data to achieve high availability, low cost, high performance, and low overhead satellite navigation system data cluster management.

(4) Data mining of Beidou panoramic mass data and regular characterization of multidimensional characteristics

Beidou data mining is the process of extracting useful information from large data sets or data streams. By mining and fusing data from different sources, you can discover the potential relationship between the data and obtain a more accurate understanding of things. Insist on observing the problem from a one-sided and isolated perspective, only like "blind people touch the elephant", and because of the limited perspective, the conclusion is biased.

By mining the Beidou panoramic data and using its redundancy and complementarity in time and space, the multidimensional regular representation of the Beidou big data can be obtained, thereby realizing real-time, continuous, macroprecision grasp of the Beidou situation, and multiangle, multilevel, and multimode accurate perception of the local system status of Beidou.

(5) Beidou multisource multidimensional big data fusion technology

The big problem that Beidou big data needs to solve now is how to use data association, crossover, and fusion to maximize the value of big data. It is believed that the key to solving this problem lies in the integration of Beidou big data, which is different from the traditional data integration or knowledge-based technology and requires a large-span, deep, and comprehensive research method. The key point is to transform the general knowledge obtained in the Beidou data fusion stage into comprehensible knowledge and provide knowledge services for demand. It needs to mine hidden knowledge, find potential knowledge associations, and then achieve a deep understanding of knowledge in order to better explain the data.

\section{(6) Multidimensional application of Beidou big data}

The big data application of Beidou focuses on the internal operation of the system and the added value added outside the system.
(1) Internal Application of Beidou Big Data System, Focusing on System Operation Management, Satellite Status Assessment Prediction, Ground Station Status Assessment Prediction, Service Performance Assessment Measurement, User Service Situation Analysis, and Other Data Applications. The internal application research work of Beidou big data system has just started, the research results are rare, there is a lack of comprehensive system research, many problems have not been solved, a large number of new problems have yet to be discovered, and research in this area needs to be urgently carried out.

(2) Additional Value-Added Services of Beidou Big Data. The added value based on the value-added data allows the big data industry to extend the extension of big data applications. In the tourism industry, Beidou, as an important means of relying on transportation, chooses to provide the tourism industry with passenger flow data and the characteristics it presents, which is convenient for the industry's profit. At the national level, the spatial and temporal characteristics based on the Big Dipper data can be used as one of the important evaluation indicators of regional economic development according to the density and flow of people. The Beidou big data value-added service will become an important part of the country's economic development in the future. The application prospect of Beidou big data will become broader as it cross-links with different industries.

\section{Big Data Technology Leads the Development of Beidou System}

The use value of Beidou big data is huge. The application of big data technology to Beidou satellite navigation system can not only further improve the analysis and processing capabilities of the system, but also raise the management and operation level of the Beidou system to a new height and explore new ways to improve the service performance of the system. The technical route has even fundamentally changed the system operation and maintenance mechanism.

First: data collection elements. It mainly refers to various navigation data sources. On the one hand, it is a data source generated within the navigation satellite system in orbit, involving spaceborne time-frequency evolution data, including frequency accuracy, frequency stability, and frequency drift rate; space atomic frequency standard transfer data; star-earth two-way time transfer comparison data; and satellite ephemeris data. On the other hand, it includes data sources generated by external real-time monitoring of aeronautical satellite systems, involving earth orientation parameters, and real-time space-based monitoring data of space environments such as the ionosphere and troposphere. The third aspect is the daily management information of the navigation satellite, such as system coordinate data.

Second: data acquisition layer. Establish a data collection network for various navigation elements. The research and 
application of Beidou big data needs to pay special attention to data collection work and needs to be classified and constructed according to different situations. Special attention should be paid to the construction of the data collection system of the navigation satellite itself, combined with the basis of traditional remote control telemetry, to upgrade the monitoring network of the on-board autonomous health management system, and special attention to the health status data collection of the sun wing, various loads, and platform information design satellite. The real-time data monitoring system outside the system strengthens the real-time collection of solar pressure and plasma data on the basis of ionospheric and tropospheric data collection.

Third: the platform storage management layer. Build a set of developed, flexible, and scalable distributed data storage management system based on Hadoop platform. Use various data collection methods to gather various data resources, establish system data application service specifications, comprehensively sort out, classify, and integrate the data collected by the navigation system, and realize a highavailability, low-cost, high-performance, and low-cost satellite navigation system data cluster management.

Fourth: data mining extraction layer. Based on big data analysis methods such as association analysis, visual analysis, knowledge mining, and data fusion, solve the data value of Beidou navigation satellite operation management, find problems, and develop key applications:

(1) In Terms of Value Discovery. This includes but not limited to the following fields, such as precision satellite delay error prediction, precision satellite clock error prediction and precision ephemeris error prediction, and star-to-earth time comparison error prediction discovery;

(2) Evaluation and early warning based on various data, such as on-plane fault correlation analysis and evaluation, ionospheric tropospheric early warning, plasma, and solar light pressure early warning

(3) Evaluation of navigation satellite performance, such as satellite monitoring and evaluation, ground station monitoring and evaluation, and system service performance monitoring and evaluation

(4) Coordinate maintenance of Beidou satellite navigation system, and the fifth layer is the system intelligent application layer. Through the research and application of Beidou big data, it helps to intelligentize system operation management, network operation management, transparency of operation management, and research and innovation model of Beidou. Build a new generation of Beidou satellite navigation system with "data support, system coordination, autonomous operation, and resource conservation."

\section{Conclusion}

Big data is an important application field of big data. On the one hand, with the construction of the Beidou satellite navi- gation system, a large amount of monitoring data has been generated. How to process these data and tap its value is a problem facing the Beidou satellite navigation system. In this paper, fog computing is illustrated in the satellite system to improve the system performance. The system's own data can also make full use of external data, vigorously improve the system's development and operation level, improve the level of Beidou satellite navigation system to serve society and service users, and expand value-added services.

\section{Data Availability}

No data were used to support this study.

\section{Conflicts of Interest}

The authors declare that they have no conflicts of interest.

\section{Acknowledgments}

This work was supported by National Natural Science Foundation of China Major Research Project under Award 91538109 and the National Nature Science Foundation of China under Award 61203226.

\section{References}

[1] D. Graham-Rowe, D. Goldston, C. Doctorow et al., "Big data: science in the petabyteera," Nature, vol. 455, no. 7209 , pp. 89, 2008

[2] H. You, Z. H. U. Yangyong, and Z. Peng, "Panorama of national defense big data," Systems Engineering and Electronics, vol. 38, no. 6, pp. 1300-1305, 2016.

[3] C. Gong, F. Lin, X. Zhou, and X. Lü, “Amygdala-inspired affective computing: to tealize personalized intracranial emotions with accurately observed external emotions," China Communications, vol. 16, no. 8, pp. 115-129, 2019.

[4] M. Wang, H. Xu, S. Yang, L. Yang, R. Duan, and X. Zhou, "Non-cooperative differential game based energy consumption control for dynamic demand response in smart grid," China Communications, vol. 16, no. 8, pp. 107-114, 2019.

[5] J. Xie, J.-j. Zhang, and G. Wang, "The construction method of BeiDou satellite navigation measurement error system," Wireless Communications and Mobile Computing, vol. 2019, Article ID 1438739, 15 pages, 2019.

[6] Z. Jianjun, J. Xie, and M. Xue, "Research on space non cooperative target of relative navigation system based on GNSS reflected signal bistatic radar.," in Proceedings of the 14th International Space Conference of Pacific-basin Societies (ISCOPS'14), Xi An, 2014.

[7] J. Su, S. Yang, H. Xu, and X. Zhou, "A Stackelberg differential game based bandwidth allocation in satellite communication network," China Communications, vol. 15, no. 8, pp. 205214, 2018.

[8] K. Xiong, P. Y. Fan, C. Zhang, and K. B. Letaief, "Wireless information and energy transfer for two-hop nonregenerative MIMO-OFDM relay networks," IEEE Journal on Selected Areas in Communications, vol. 33, no. 8, pp. 11611, 2015.

[9] K. Xiong, C. Chen, G. Qu, P. Fan, and K. B. Letaief, “Group cooperation with optimal resource allocation in wireless 
powered communication networks," IEEE Transactions on Wireless Communications, vol. 16, no. 6, pp. 3840-3853, 2017.

[10] T. Lv, H. Gao, and S. Yang, "Secrecy transmit beamforming for heterogeneous networks," IEEE Journal on Selected Areas in Communications, vol. 33, no. 6, pp. 1154-1170, 2015.

[11] A. Hu, T. Lv, H. Gao, Z. Zhang, and S. Yang, “An ESPRITbased approach for 2-D localization of incoherently distributed sources in massive MIMO systems," IEEE Journal of Selected Topics in Signal Processing, vol. 8, no. 5, pp. 9961011, 2014. 